\title{
RESISTANCE OF METALLIC SUBSTRATES PROTECTED BY AN ORGANIC COATING CONTAINING GLASS FLAKES
}

\author{
J. González-Guzmán, J.J. Santana ${ }^{+}$, S. González and R.M. Souto \\ Department of Physical Chemistry, University of La Laguna, \\ E-38071 La Laguna, Tenerife, Canary Islands, Spain.
}

\begin{abstract}
:
The resistance against corrosion of an epoxy-polyamine-based coating immersed in a $3 \mathrm{wt} \%$ sodium chloride solution was investigated by electrochemical impedance spectroscopy (EIS). The organic coating contained glass flakes as pigment in order to enhance its barrier characteristics. The data show that this coating is more strongly adhered and exhibits higher protection characteristics when applied onto carbon steel substrates than on galvanized steel. Though the capacitance of the coating $\left(C_{C}\right)$ does not show any appreciable variation with immersion time, the resistance $\left(R_{P O}\right)$ of the film is observed to increase with time upon immersion. The analysis of the data sustains that the organic film behaves as a porous, non-barrier coating. Two time constants are observed even at earlier exposures, and the improved corrosion resistance developed after the coating system was exposed to the test electrolyte is considered to originate from the precipitation of corrosion products within the pores in the film.
\end{abstract}

Keywords: Organic coatings; Corrosion prevention; Electrochemical impedance spectroscopy; Glass flake pigments; Epoxy-polyamine.

+ On leave from the Department of Process Engineering, University of Las Palmas de Gran Canaria, Campus Universitario de Tafira, E-35017 Las Palmas de Gran Canaria, Canary Islands, Spain. 


\section{Introduction}

Organic coatings are the most widely used method of protection against atmospheric corrosion of metals. The paint film acts as a barrier between the aggressive environment and the metallic material, though the coating is permeable to aggressive species such as water and oxygen [1-5]. New coatings are developed that may more effectively withstand the many aggressive environments metallic materials are exposed during operation. In particular, pigmented paint systems are used because they exhibit an enhanced protectiveness against corrosion. Additives may achieve such effect either by exhibiting active barrier properties, or by affecting the corrosion reactions responsible for the degradation of the metal-coating system. Thus, active pigments may electrochemically protect the metallic substrate through galvanic or passivation mechanisms, or they may contribute to the barrier properties of the coating through the chemical precipitation of solid compounds. In recent years, anticorrosive paints containing glass flakes are being investigated in relation to metal protection in highly corrosive media because they are expected to greatly hinder the water transport through the polymeric matrix.

The purpose of the present study was to investigate the corrosion behaviour of a commercial paint system that contains glass flakes as pigment. The binder was an epoxy-polyamine resin. The substrates selected for this work were a carbon steel and a galvanized steel. Electrochemical impedance spectroscopy (EIS) was used to measure the resistance of painted samples after exposure to $3 \% \mathrm{NaCl}$ aqueous solution, and the impedance data were analysed in terms of the timedependency of characteristic parameters [6-10]. Next, impedance data were combined with data from pull-off adhesion tests to investigate the extent to which differences in the adhesion between the organic coating and the underlying metallic substrates affected the corrosion resistance of these materials.

\section{Experimental details}

Carbon steel and galvanized steel plates were employed as metallic substrates. The thickness of the Zn layer was $20 \pm 2 \mu \mathrm{m}$. Metal surfaces were carefully washed in acetone, ethanol and doubly distilled water, and then dried prior to the coating process. The samples were painted in the laboratory to a thickness of $60 \pm 2 \mu \mathrm{m}$ with a withdrawing technique using an accurate speed motor and a system of gears. Coated specimens were allowed to cure at room temperature and humidity for 
five days prior to testing. The coating was a two component epoxy-polyamine resin pigmented with glass flakes as pigment (SIGMACOVER TCP GLASSFLAKE) supplied by Sigma Coatings (Amsterdam, The Netherlands). The paint was prepared in the laboratory in order to control the quality of the coatings. The film's thickness was measured with a Mega-Check FN CoatingThickness-meter (List-Magnetik GmbH, Germany).

The test solution was a $3 \mathrm{wt} . \% \mathrm{NaCl}$ aqueous solution, open to air, held at room temperature. The time of exposure was ca. 400 days. A three-electrode cell arrangement was used in the experiments. The electrochemical cell was a flat-cell provided with a sufficiently large stainless steel counter electrode that was positioned parallel to the $13 \mathrm{~cm}^{2}$ working electrode surface. A saturated calomel electrode (SCE) was employed as reference electrode.

Electrochemical impedance spectroscopy data were obtained using a computer-controlled EG\&G Model 5210 lock-in amplifier connected with an EG\&G Model 283A potentiostat. The impedance measurements were carried out over a frequency range of $10 \mathrm{kHz}-1 \mathrm{mHz}$ using a $15 \mathrm{mV}$ amplitude of sinusoidal voltage. The data quality acquisition was established in four cycles at each frequency, thus providing good signal-to-noise ratios at all frequencies. The software used to acquire and analyse the data allowed representation of the data in terms of both Bode (logarithm of the impedance modulus $|Z|$ and phase angle $\Phi$ as a function of the logarithm of the frequency $f$ ) and Nyquist (imaginary component of the impedance as a function of the real component) plots. The impedance spectra were analyzed using Yeum’s “ZsimpWin 2.00” software [11].

The initial adhesion and the adhesion retained after immersion in $3 \% \mathrm{NaCl}$ aqueous solution were measured using a direct pull-off adhesion test. In this method, the test dolly was bonded to the coating using an appropriate adhesive (Araldite $\left.{ }^{\circledR}\right)$. The samples for retained adhesion studies were removed from the solution at the end of the immersion test, rinsed thoroughly with distilled water and allowed to dry for 48 hours at ambient temperature. A digital adhesion tester AT-1 (Neurtek, Spain) with a maximum applied load of $160 \mathrm{~kg} \mathrm{~cm}^{-2}$ was employed.

\section{Results and discussion}

Measurements of electrochemical impedance have been carried out at the open circuit potential $\left(E_{c o r r}\right)$ after immersion for different times in $3 \% \mathrm{NaCl}$ solution, at room temperature. Typical examples of the Nyquist and Bode impedance plots obtained for the coated samples are shown in figures 1 and 2. They were obtained for the two metallic substrates under consideration. 
Changes in the impedance characteristics as a result of the exposure of the specimens to the corrosive environments could be deduced from the comparison of the spectra. From a cursory observation of these plots it can be deduced that two time constants are operating in these systems at all times. Thus, the spectra could be analysed in terms of the equivalent circuit for a defective organic coating $[18,19]$ that is depicted in figure 3. It assumes that the corrosion of the coated metal is controlled by the same mechanism that occurs on the bare metal but with a greatly reduced active surface area. The equivalent circuit consists of the resistance $R_{\Omega}$ of the test electrolyte and electrical connectors, the capacitance $C_{C}$ of the intact coating layer, the pore resistance $R_{P O}$ due to penetration of electrolyte, the polarization resistance of the substrate $R_{t}$, and the double-layer capacitance at the substrate/electrolyte interface $C_{D L}$.

Analysis of the impedance spectra in terms of this equivalent circuit allowed for the parameters $C_{C}$ and $R_{P O}$ to be determined. Changes in the magnitudes of these parameters as a function of the immersion time in the test solution for the various coatings and substrates are plotted in figures 4 and 5. The comparison of the values of these parameters as a function of time should provide information regarding their protective abilities to hinder the corrosion of the underlying metal in the corrosive medium. From the inspection of the graphs in figure 4, the values of the coating capacitance $C_{C}$ were regarded to remain almost invariant with time, and all the values were in the order of $10^{-9} \mathrm{~F} \mathrm{~cm}^{-2}$, which are typical of a barrier coating with dielectric characteristics. Therefore, no relevant conclusions concerning the effectiveness against corrosion of the coated systems could be extracted from the time course of the coating capacitance this time.

On the other hand, it was observed that the resistance of the coating remained moderately high ( $>10^{7} \Omega \mathrm{cm}^{2}$ ) for the total exposure time of almost 9600 hours. In general, RPO are almost one order of magnitude greater in the case of the coating applied on carbon steel, and they are found to drastically increase during the first week of exposure, though they continued steadily growing for about 330 days (see figure 5A). The first stages of further coating degradation might be evidenced by the small decrease in $R_{P O}$ values at the longest exposures, though a drastic decrease in the values that could be related to failure of the film was not found until 400 days immersion in the aggressive environment.

The time course of $R_{P O}$ is less regular in the case of the coating applied on galvanized steel, as depicted in figure 5B. The initial trend to growth of the values is broken after 3 weeks, and it only resumes after 2 months. Even then, the growth is rather small and not sustained for the remaining experiment. 
An alternate route for the evaluation of the corrosion resistance of coated metals is provided by means of the break-point frequency method [14-16], which consists in the analysis of the time course of the characteristic breakdown frequency for each impedance spectrum. This characteristic breakdown frequency, $f_{b}$, is the frequency at which the phase angle $\Phi=45^{\circ}$ in the capacitiveresistive transition region. The break-point frequency is related to the delaminated area [15], and gives a measure of the ratio between the damaged and intact coating areas as:

$$
f_{b}=\frac{A_{d}}{2 \pi \varepsilon \varepsilon_{0} \rho_{0} A}=\frac{K A_{d}}{A}
$$

where $A_{d}$ is the delaminated area, $A$ is the total area of the sample, $\varepsilon_{0}$ is the vacuum permittivity, $\varepsilon$ is the dielectric constant of the organic coating, and $\rho_{0}$ is the specific resistivity of the coating in the delaminated area. Thus, the trends in figure 6 should qualitatively represent the time course degradation of the coated materials. No clear breakdown of the coated films was observed for the duration of the experiments, as it would be characterized by a drastic increase in the values of the breakdown frequency for a given system. Neither such a drastic decrease is observed in $R_{P O}$ values for any of the samples (cf. figure 5). In general, direct comparison of the breakdown frequency values displayed in figure 6 allow to conclude that the highest anticorrosion resistance is displayed by this coating when it is applied on carbon steel, whereas the less resistant systems occurred on galvanized steel. It should also be noticed that the values show a steady trend to decrease throughout the experiment in the case of the coating applied on carbon steel (see figure 6A), a trend that is only interrupted for exposures in excess of 330 days.

In order to explore the effect of the adhesion between the organic coating and the metallic layer may be also relevant in this case, pull-off tests were performed both prior and after the immersion tests, and the results are given in Table 1. In general, the adhesion decreased after the corrosion tests for all the samples, though the adhesion of the polymeric film to carbon steel was significantly greater than for galvanized steel, this effect being more notorious for the coating applied on carbon steel. Yet, the force employed to detach the degraded coating on carbon steel after 400 days exposure was indeed greater than that for the original coating applied on galvanized steel. This may suggest that, for a porous film such as this epoxy-polyamine matrix under investigation, the corrosion reactions within the pores might lead to a significantly greater attack in the case of the galvanized steel, with the result of a delamination front to develop from the pore due to the weaker adherence of the film. In these circumstances, though the precipitation of zinc corrosion products might produce a greater resistance for the corrosion reaction to progress, the effect would be hardly noticeable from the time courses of both the coating resistance, $R_{P O}$, and the corresponding 
breakdown-point frequencies, $f_{b}$, in the spectra (cf. figures 5B and 6B). Conversely, the corrosion products formed can rather effectively block the narrow pores in the case of the coating applied on carbon steel. In fact, a rather peculiar behaviour of the breakdown frequency values with the elapse of time is found (see figure 6A), which would correspond to a decrease of the delaminated area with the elapse of time when the metal-coating systems was immersed in the aggressive electrolyte.

\section{Conclusions}

EIS measurements have shown the characteristic behaviour towards corrosion protection of steel substrates exhibited by an epoxy-polyamine coating containing glass flake additions. EIS data support that this primer is more indicated for the protection of carbon steel since the barrier characteristics of the film against water transport provide corrosion protection to the underlying metal. Less satisfactory results were found after the application of the primer on galvanized steel. Thus, the nature of the underlying metallic substrate is a key factor affecting the efficiency of a given coating system towards corrosion protection in addition to surface preparation of the metal.

The coating system under investigation originates a polymeric film which is quite rough in its surface, and micropores may be left through the polymer matrix during the curing stage. This observation is consistent with the observation of two time constants in the impedance spectra, which effectively corresponds to a non-barrier film, and the onset of corrosion reactions in the pores. Thus, the corrosion resistance of the metal-coating system mainly arises from the capacity of corrosion products to block the micropores through precipitation. The application of more than one layer of the coating would be then required to provide effective corrosion protection, especially for the protection of galvanized steel substrates.

Since the strength of adhesion between the polymer film and the underlying metal is significantly higher when it was applied on carbon steel, may also be related to the better corrosion protection characteristics of this metal-coating system, as it has been previously observed for other systems $[8,10]$.

\section{Acknowledgements:}


This work was supported by the Ministerio de Ciencia y Tecnología (Madrid, Spain) under Project No. CTQ2005-06446. A grant awarded to JJS by the Gobierno de Canarias (Spain) to conduct a research stay at the University of La Laguna is gratefully acknowledged. Thanks are due to Sigma Coatings (Amsterdam, The Netherlands) for providing the coatings.

\section{References:}

1. H. Leidheiser, Jr., Corrosion 38 (1982) 374-383.

2. G.W. Walter, Corrosion Science 26 (1986) 27-38.

3. G.W. Walter, Corrosion Science 32 (1991) 1041-1058.

4. L.L. Shreir, R.A. Jarman and G.T. Burstein (Eds.), Corrosion, 3rd. edn., Vol. 2. ButterworthHeinemann, Oxford (1994), Ch. 14.

5. E.P.M. van Westing, G.M. Ferrari and J.H.W. de Wit, Corrosion Science 36 (1995) 957-977.

6. R.M. Souto, V. Fox, M.M. Laz and S. González, Journal of Adhesion Science and Technology 14 (2000) 1321-1330.

7. S. González, M.A. Gil, J.O. Hernández, V. Fox and R.M. Souto, Progress in Organic Coatings 41 (2001) 167-170.

8. Y. González-García, S. González and R.M. Souto, Journal of Adhesion Science and Technology 19 (2005) 1141-1155.

9. R.M. Souto, L. Fernández-Mérida, S. González and D.J. Scantlebury, Corrosion Science 48 (2006) 1182-1192.

10. Y. González-García, S. González and R.M. Souto, Corrosion Science, 49 (2007) 3514-3526.

11. B. Yeum, Electrochemical Impedance Spectroscopy: Data Analysis Software. Echem Software, Ann Arbor (2001).

12. F. Mansfeld, Journal of Applied Electrochemistry 25 (1995) 187-202.

13. M. Kendig, S. Jeanjaquet, R. Brown and F. Thomas, Journal of Coatings Technology, 68 (863) (1996) 39-47.

14. S. Haruyama, M. Asari and T. Tsuru, in: Corrosion Protection by Organic Coatings, M.W. Kendig and H. Leidheiser, Jr. (Eds.). The Electrochemical Society, Pennington, NJ (1987), pp. 197-207.

15. R. Hirayama and S. Haruyama, Corrosion 47 (1991) 952-958.

16. N.J. Koulombi and S.T. Kyvelidis, Mikrochimica Acta 136 (2001) 175-180. 
Table 1. Pull-off test results. The data are expressed as the forces required to detach the film from the metallic substrate. The values are expressed in $\mathrm{kg} \mathrm{cm}^{-2}$.

\begin{tabular}{|c|c|c|}
\hline SAMPLE CHARACTERISTIC & non-exposed & after exposure \\
\hline $60 \mu \mathrm{m}$ thick coating on carbon steel & 42.0 & 30.4 \\
\hline $60 \mu \mathrm{m}$ thick coating on galvanized steel & 25.1 & 20.5 \\
\hline
\end{tabular}

Figure 1 - Experimental impedance spectra of a carbon steel coated with an epoxy-polyamine film

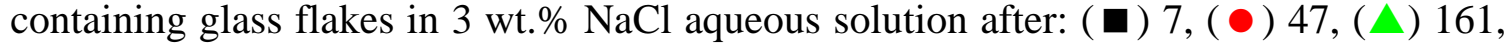
$(\diamond)$ 310, and $(\star) 361$ days. Coating thickness: $60 \mu \mathrm{m}$.
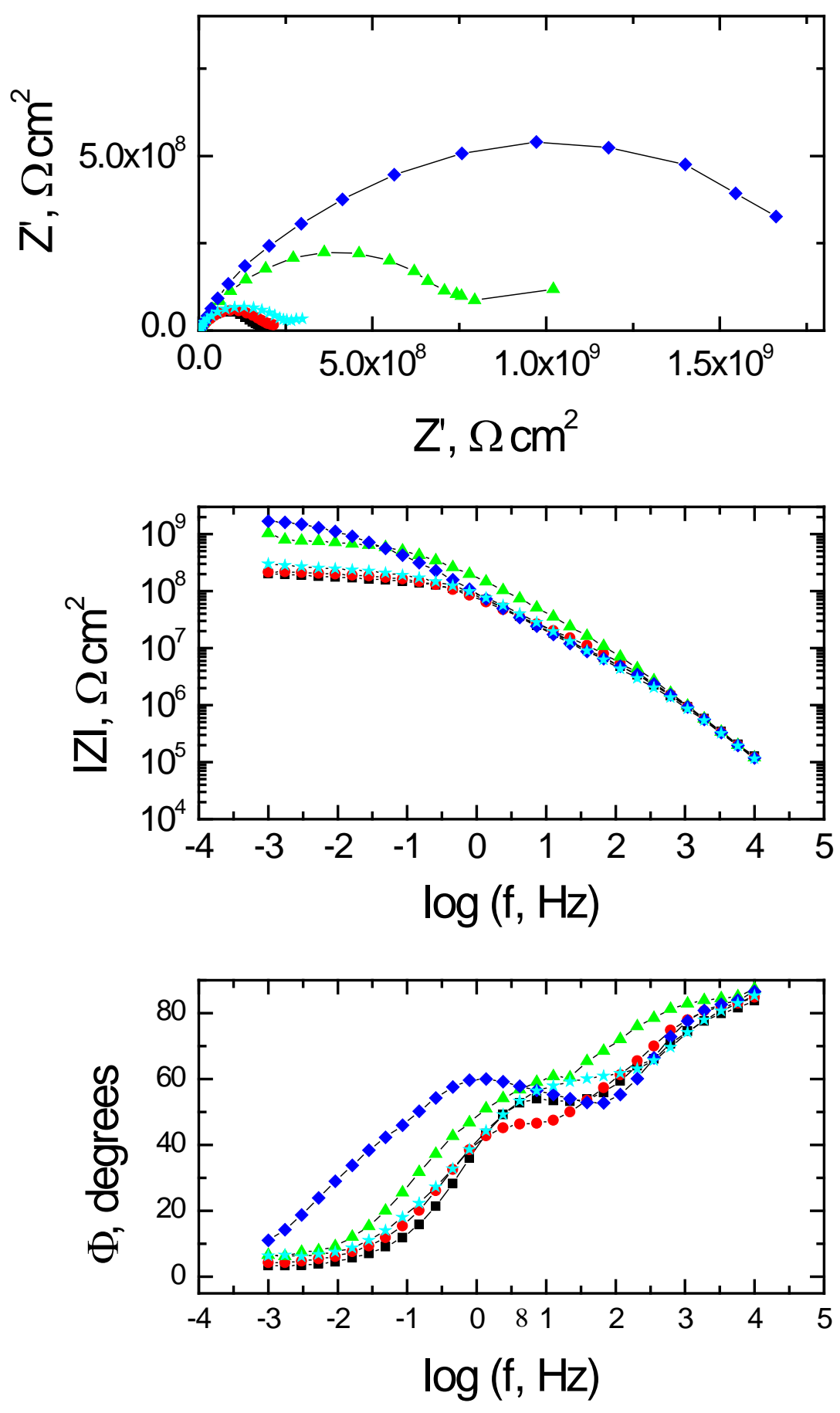
Figure 2 - Experimental impedance spectra of a galvanized steel coated with an epoxy-polyamine film containing glass flakes in 3 wt.\% $\mathrm{NaCl}$ aqueous solution after: $(\boldsymbol{\square}) 7,(\bullet) 23,(\triangle)$ 39, $(\diamond)$ 121, ( ) 185, ( ) 235, and ( 345 days. Coating thickness: $60 \mu \mathrm{m}$.
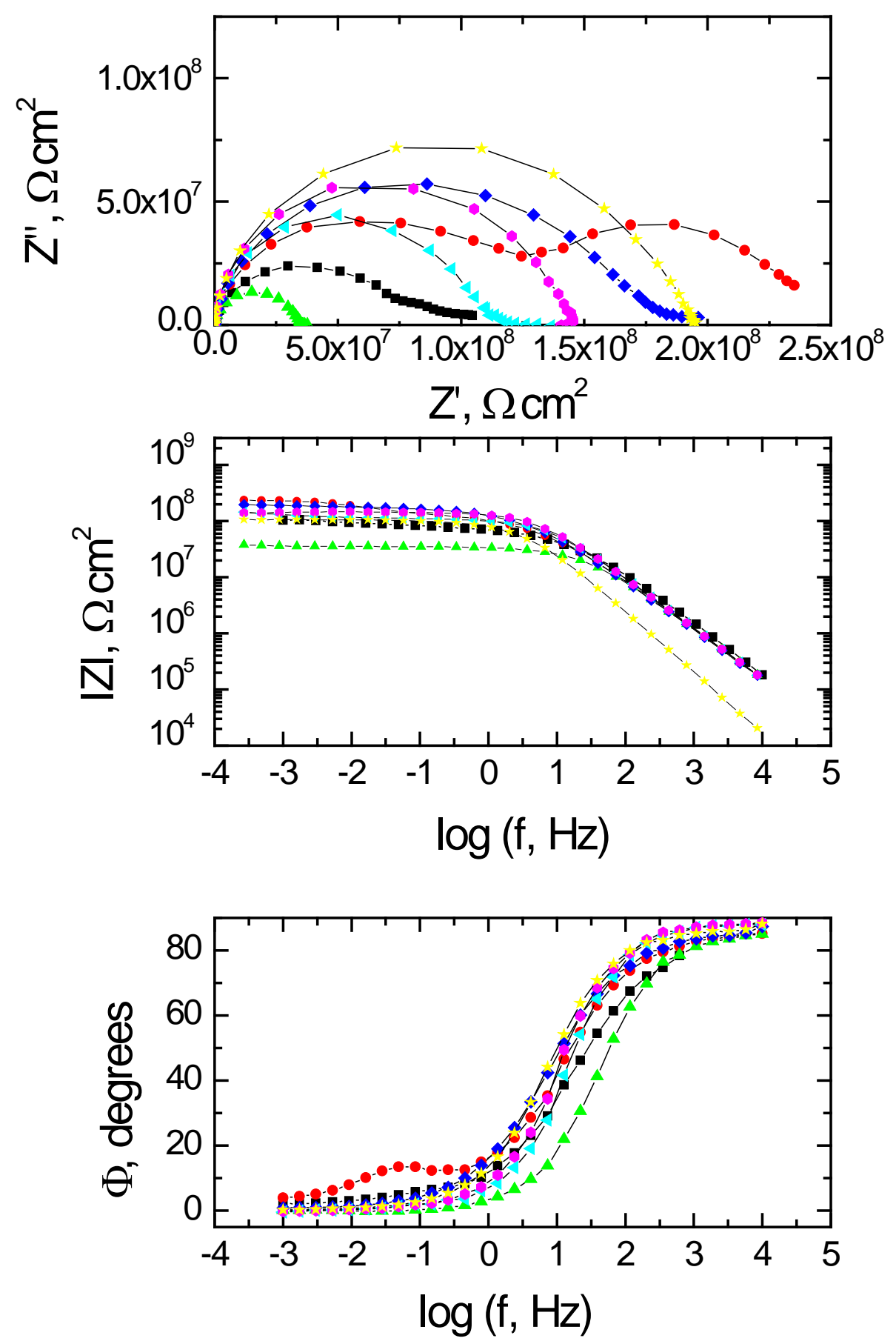
Figure 3 - Equivalent circuit for the description of a non-barrier coating system with two time constants [12,13].

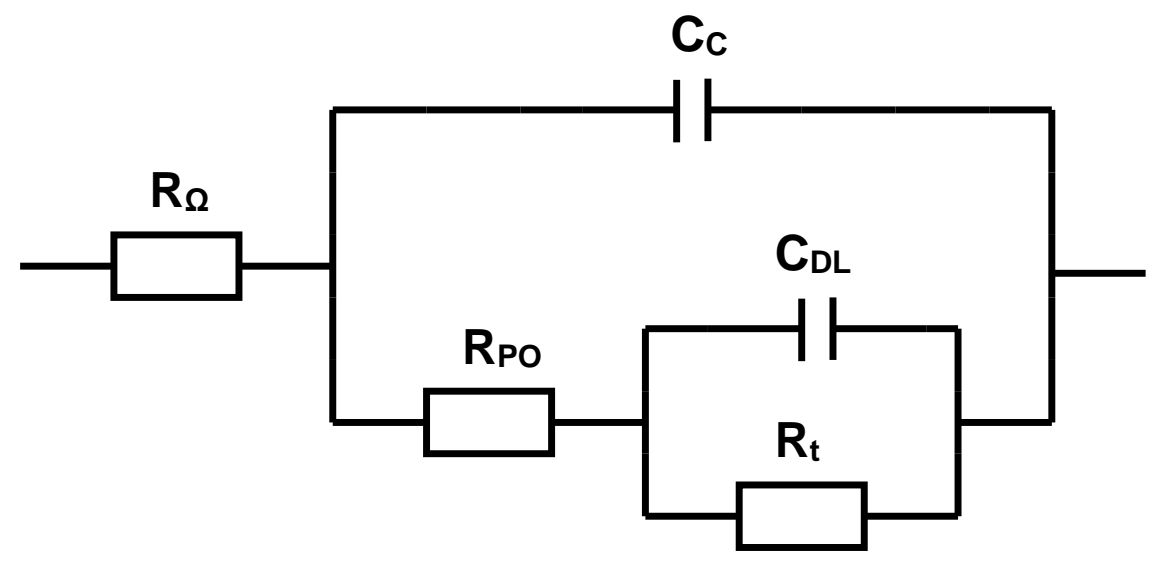

Figure $4-C_{C}$ values as a function of exposure time for glass flake containing epoxy-polyamine coated specimens. (A) carbon steel coated with a $60 \mu \mathrm{m}$ film; and (B) galvanized steel coated with a $60 \mu \mathrm{m}$ film.

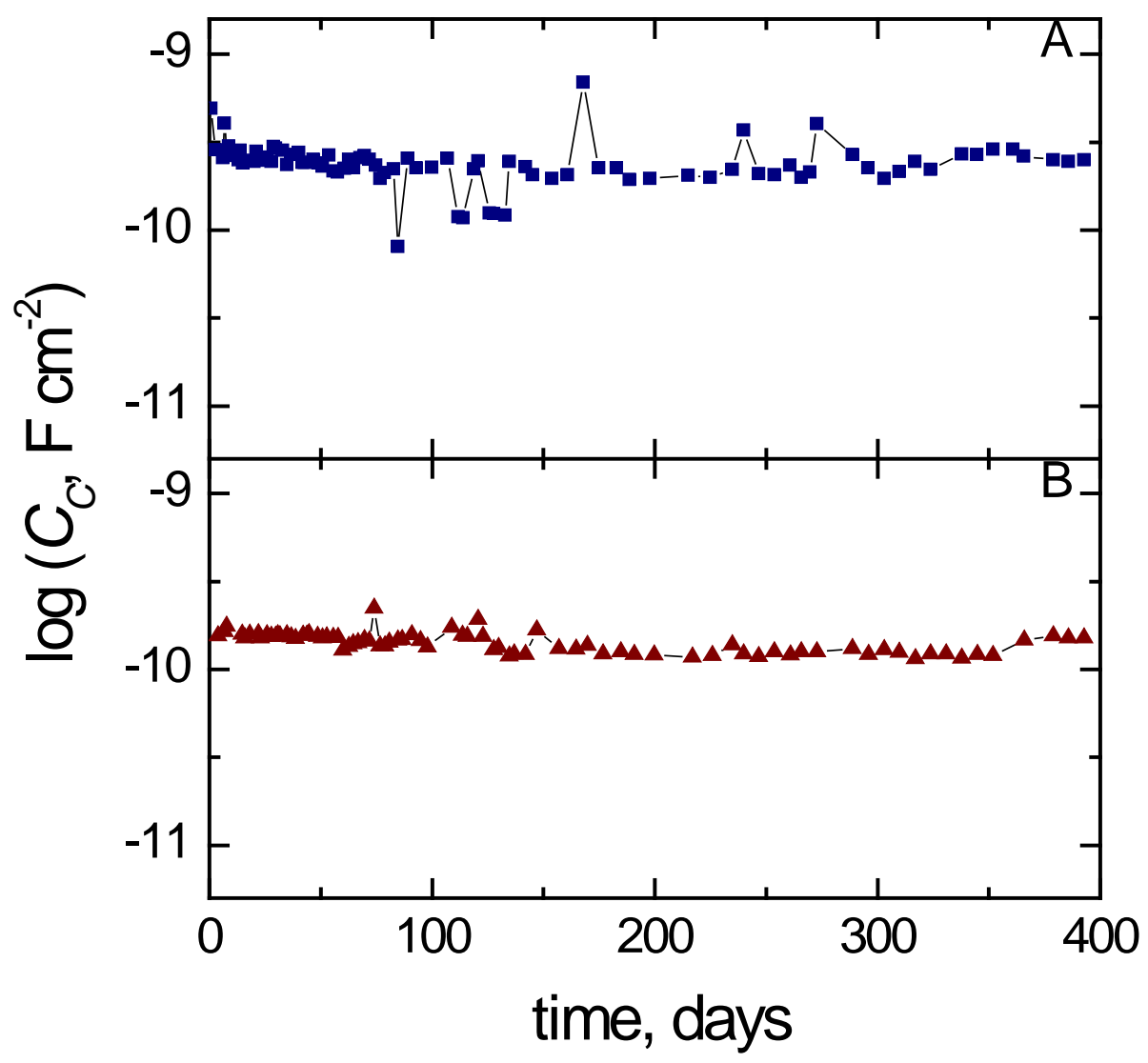


Figure 5 - $R_{P O}$ values as a function of exposure time for glass flake containing epoxy-polyamine coated specimens. (A) carbon steel coated with a $60 \mu \mathrm{m}$ film; and (B) galvanized steel coated with a $60 \mu \mathrm{m}$ film

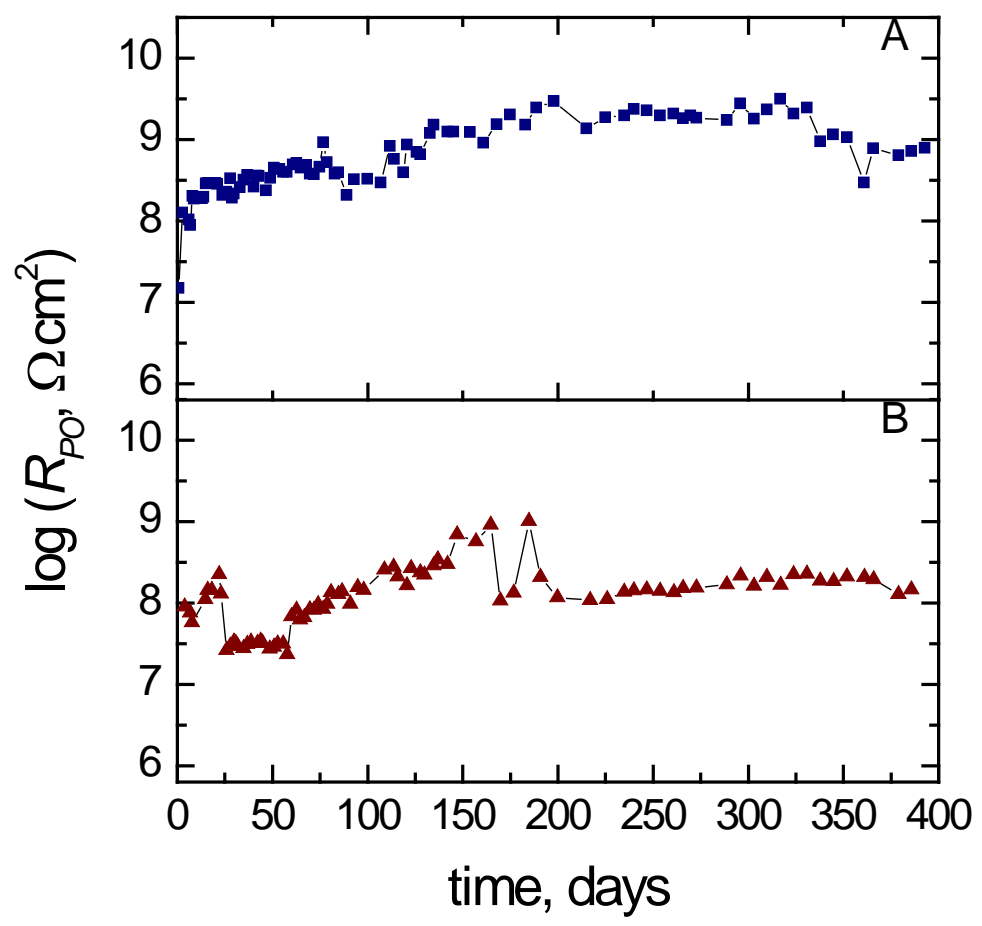

Figure $6-f_{b}$ values as a function of exposure time for glass flake containing epoxy-polyamine coated specimens. (A) carbon steel coated with a $60 \mu \mathrm{m}$ film; and (B) galvanized steel coated with a $60 \mu \mathrm{m}$ film.

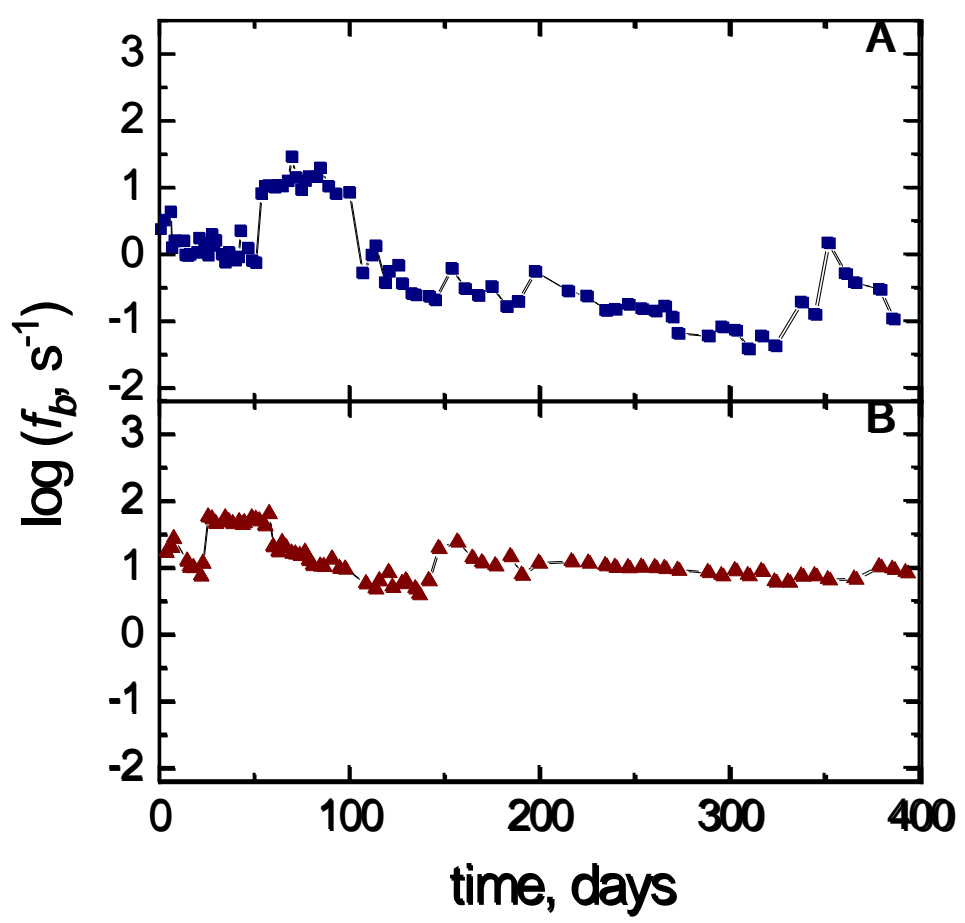

